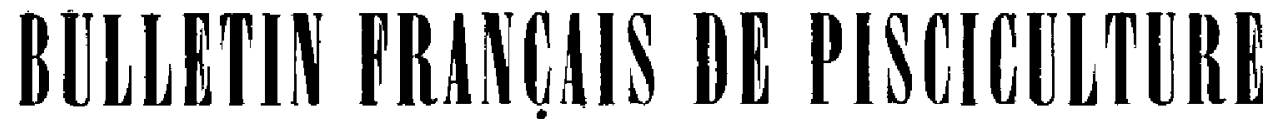

\section{NOT E \\ sur le fonctionnement du Clapet automatique AVEQUE, par l'action conjointe du flux maritime à l'aval et d'une crue fluviale à l'amont}

par M. ANDRÉ GARDEBOIS

Architecte A. P., à Meudon (Seine-et-Oise)

Les fleuves cotiers du Nord et du Nord-Ouest de la France sont, pour lia plupart, remontés pár les grands Salmonides, et il est nécessaire, pour favoriser leur migration, de pourvoir, d'une échelle à poissons, les barrages édifiés sur ces cours d'eau.

Ce dispositif a fait ses preuves depuis que le coursier est muni :

$I^{\circ}$ Des amortisseurs brevetés conçus par M. I'Inspecteur Généra' des Eaux el Forèts de Lachadexìde, destinés à freiner le courant et à le maintenir à une vitesse inférieure à la vitesse de nage des poissons lors de leur remontée ;

$2^{\circ}$ Du clapet automatique inventé par M. Aveque (i) qui permet l'admission de l'eau dans l'áchelle en période de crre seulement, c'est-à-dire quand l'eau dépasse la crête du déversoir. Le fonctionnement de l'échelle, dont le débit nécessaire est de I.200 à I.joo litres secondes, pour une largeur de $1 \mathrm{~m}$. 5o, ne porte done ancun préjudice anx utilisateurs de l'énergie hydraulique.

L'emploi maximum de celte énergie sur les fluves côtiers a incité à multiplicr les barrages, sur leurs cours ; par ailleurs, les nécessités de la navigation ont amené à édifier des ouvrages de retenue.

En consćquence, un certain nombre de barrages ont été construits à proximité des estuaires où ils se trouvent ainsi soumis à l'actioṇ des marées : tels sont ceux de la Comparnie des Anciens Etablissements de la Risle, à Pont-tudemer, sur la Risle, de Caen, sur l'Orne, de Saint-Brieuc sur le Gouet, etc...

(1) Nous tenons à rendre hommage à M. Aveque quí a conçu et réalisé le clapet automatique qui porte son nom, objet de cette modeste étude, qu'il a bien voutu examiner et compléter par une suggestion élégante... 
Or, il a été observé que les migrateurs suivent la marée et remontent les estuaires avec le flux. Il est sonc suffisant de n'alimenter les échelles que l'on se propose d'édifier qu'en hautes eaux.

Il y a, à cela, plusieurs avantages :

I $^{\circ}$ L'extrémité aval de l'échelle pourra être exhaussée et établie à une cote correspondant aux hautes eaux, diminuant ainsi la hauteur à gravir ;

$3^{\circ}$ L'échelle sera moins longue el l'effort à fournir par les migrateurs lors de leur remontée sera réduit. En conséquence, l'aménagement d'un bassin de repos sera rendu inutile;

$3^{\circ}$ La réduction de longueur permettra de réaliser une économie considérable sur le cube de béton, la quantité de liant nécessaire et le nombre des éléments amortisseurs. Economie appréciable en période de pénurie de matériaux ;

$4^{\circ}$ Enfin, le cout de construction de louvrage sera nduit dans une proportion importante.

Il convient donc de chercher un dispositif capable de commander le clapet automatique Aveque, à l'aide des variations de niveau à l'aval.

Toutefois, un correctif devra être apporté. En effet, si le clapet fonctionne sous l'action des hautes eaux seules, il s'ouvrira en moyenne deux fois par 24 heures jo minutes, et il livrera passage à l'eau dans i'échelle, même en période de basses eaux à l'amont.

Il faut donc examiner si le clapet Aveque est capable, tel qu'il a été conçu, de ne faire fonctionner l'échelle qu'à marée haute à l'aval, et seulement en période de crue à l'amont du barrage.

On sait que le clapet automatique Aveque (Planche II), se compose essentiellement d'un volet ou clapet oscillant sur l'axe inférieur horizontal auquel il est fixé. Il est placé à la partie amont de l'échelle et est relié par son axe à une bielle articulée sur une tige supportant un flotteur en tôle se déplaçant verticalement dans un puits alimenté en eau, seulement quand le niveau amont dépasse la crète du déversoir du barrage.

Un orifice de vidange du puits vers l'aval est établi et l'excès d'alimentation sur l'évacuation, qui permet le remplissage du puits, cetle dernière réglée par une vannctle, assure la montée du flotteur et l'ouverture du clapet.

Si le débit de l'orifice de vidange est supérieur au débit de l'alimentation, le puits se vide, le flotteur descend, entraîné par son poids, et le clapet se ferme.

On assurera donc le fonctionnement du clapet par les variations de niveau à l'aval en mettunt le puits en communication avec le bief inférieur tu barrage soumis à l'action de la marée montante, mais comme il a été dit ci-dessus, la quantité d'eau de mer à admettre dans le puits du flot- 
- I5g-

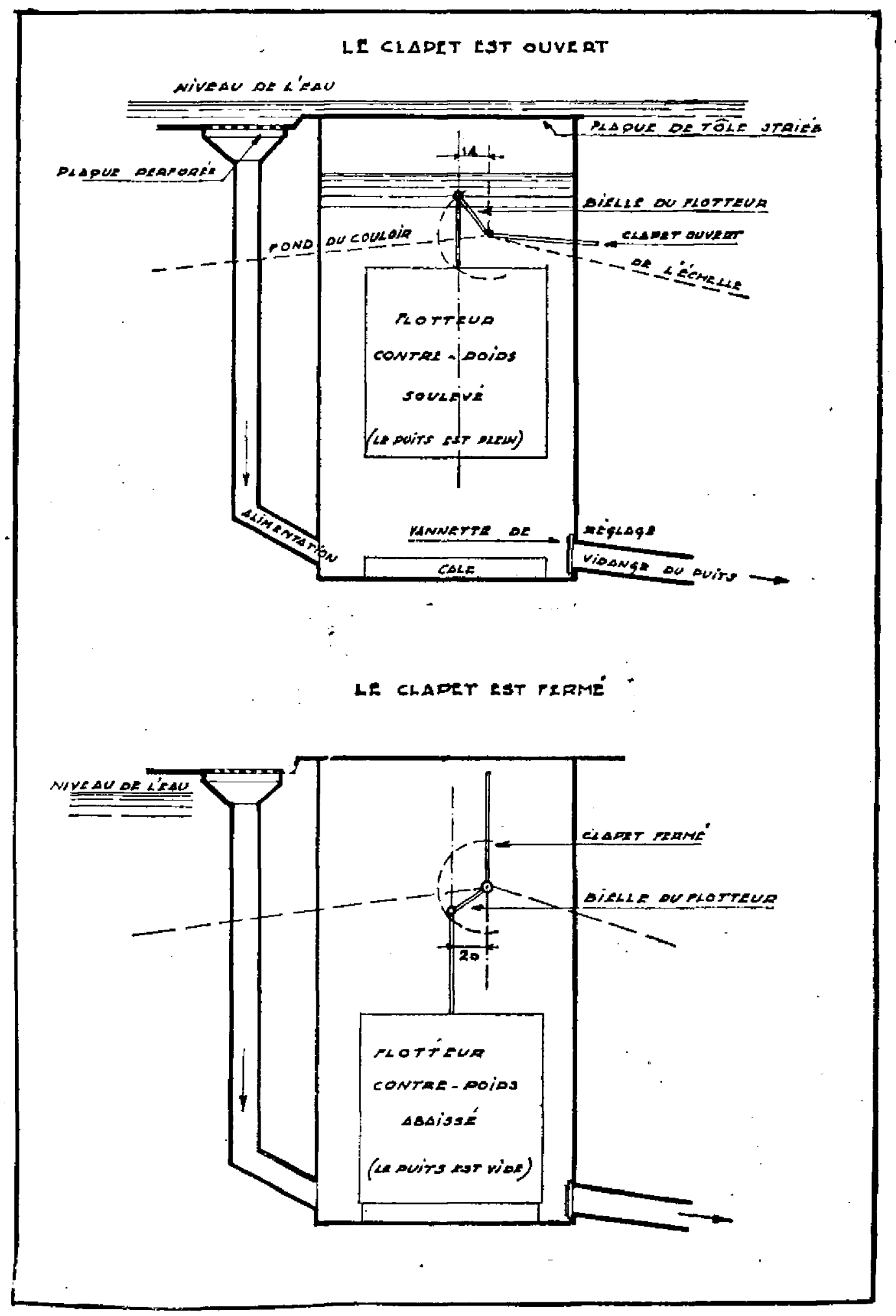

Prancrz II. - Clapet automatique Avequz. 
teur devra être insuffisante pour permettre à elle seule la montée de ce dernier et, partant, l'ouverture du clapet.

Le puits du fotteur sera, comme dans le dispositif habituel, en communication avec le bief amont du barrage, mais, en cas de crue à l'amont, la quantité d'eau à admettre dans le puits du flotteur devra c̀tre inférieure au débit de l'évacuation et insuffisante pour permettre à elle seule la montée de ce dernier et, partant, l'ouverture du clapet.

Par contre, la quantité d'eau admise dans le puits du flotteur par la marée haute à l'aval et la quantité d'eau admise à l'amont par une crue, devront étre suffisantes, en s'additionnant, pour permettre l'élévation du flotteur et l'ouverture du clapet.

En conséquence, chaque marée montante, en période de crue à l'amont, fera ouvrir le clapet de l'échelle, qui sc fermera à marée descendante.

De mème, une crue subite en marée montante provoquera l'ouverture du clapet, et la fin de cette crue, en marée montante, assurera la mise à sec de l'échelle.

Le fonctionnement. du clapet a été calculé par $M$. Aveque de taçon telle, que le flotteur en tôle qui mesure $1.10 \times$ r $.00 \times 0.90$ et pèse 800 kilogs, y compris le poids de l'eau qui y a té introduit, engendre sur l'axe du clapet, par l'intermédjaire de la biellè, quand il repose sur le radię du puits, ce dernier étant vide, un effort de

$$
800 \text { kilogs } \times 0,20=160 \text { kilogrammètres }
$$

supérieur à la pression de l'eau sur le clapet qui mesure $\mathrm{I} .50 \times 0.65$, pression égale à

$$
1.50 \times 0.65 \times 1 / 3-316 \mathrm{~kg} 875 \times 0.65 \mathrm{hr} / 3=69 \text { kilogrammètres. }
$$

Le poids du flotteur reposant sur le fonds du puits vide maintient donc le clapet fermé, l'écart entre les deux forces antagonistes étant de :

$$
160-69=9 \text { I kilogrammètres. }
$$

Si le puits sc remplit d'eau, l'éliage s'ćlevant à l'amont, et si le niveau vient à dépasser, dans le puits, la face supérieure du flotteur, qui est ainsi noyé, le volume de ce dernier étant de I. ro $\times$ I.000 $\times 0.90-0 \mathrm{mc} .990$, il perd un poids de $990 \mathrm{kilog}$.; il possède alors une force ascensionnelle de 990 kilog. -800 kilog. = I go kilogs.

Ie moment engendré par ladite force ascensionnelle, par l'intermédiaire de la bielle quand le flotteur repose au fond du puits est alors de : 190 kilogs $\times 0.20=38$ kilogrammètres.

Cette force s'ajoute à la pression exercée sur la face amont du clapet en position de fermeture, qui est de 69 kilogrammètres, ainsi que M. Aveque l'a indiqué plus haut, la poussée est donc de $69+38=107$ kilogrammètres, et le clapet oscille sur son axe en s'inclinant vers l'aval.

Quand il accupe sa position horizontale d'ouverture, il agit sur son axe 
par son propre poids, compte tenu du poids du volume d'eau qu'il déplace, poids qui engendre sur son axe un moment de :

$$
80 \text { kilogs } \times 0.65 \mathrm{hr} / 2=26 \text { kilogrammètres }
$$

l'axe est ainsi soumis à un effort de :

$$
38 \mathrm{kgm}+26 \mathrm{kgm}=64 \text { kilogrammètres. }
$$

L'effort ascensionnel du flotteur, par l'intermédiaire de la bielle, quand le flotteur est à fond de course ascensionnelle, est de :

I go kilogs $\times 0.14=26.6$ kilogrammètres.

Le clapet est donc maintenu ouvert par la force ascen-

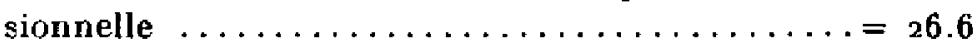

et l'effort sur l'axe du clapet horizontal. . . . . . $=26$

soit au total. . . . $=\overline{52.6}$ kilogrammètres sensiblement égal à celui du clapet fermé.

Si le niveau de l'eau baisse à l'amont, le puits se vide, le floteur, à fond de course ascensionnelle, agit alors comme contrepoids de 800 kilogs en engendrant sur son axe, par l'intermédiaire de la bielle, un effort de :

800 kilogs $\times 0.14-112$ kilogrammètres.

Le clapet en position d'ouverture et horizontal, agit en sens inverse de l'effort du flotteur, et nous avons vu que cette force, maintenant antagoniste, est de :

80 kilogs $\times 0.65 / 2=26$ kilogrammètres

l'effort de fermeture développé par la traction du flotteur est alors de :

$$
112-26=86 \text { kilogrammètres }
$$

et le clapet se ferme petit à petit, jusqu'à l'instant où, étant en position de fermeture, et le flotteur reposant au fond du puits vide, il subit une pression d'eau à l'amont de 69 kilogs inférieure à l'effort développé par le flotteur qui est de 160 -kilorrammètres.

Mais les mouvements d'ouverture et de fermelure du clapet, tels qu'ils sont exposés ci-dessus, se produiraient lentement, et sous certaines conditjons de niveau à l'amont, il s'établirait un équilibre entre la quantité d'eau admise dans le puits et la quantité d'eau évacuée. Equilibre entre les débits qui se traduirait par une ouverture ou une fermcture incomplètes du clapet assurant un débit insuffisant dans le couloir de l'échelle.

Pour éviter cet inconvénient, M. Areque a éludié un dispositif, original et ingénieux, qui permet un fonctionnement "tout ou rien ".

Ainsi qu il a élé dit ci-dessus, le clapet en tôle a une largeur identique à celle de l'échelle qu'il obture, soit $1 \mathrm{~m}$. 5o et une hauteur de 0.65 . Il est fixé par sa partie inférieure à un axe en acier de 68 millimètres de diamètre, supporté par trois paliers avec coussinets en bronze, axe pro- 
longé à l'intérieur du puits. Dans la partie de l'axe située dans le puits, une bielle est clavetée à l'une de ses extrémités et reçoit, à l'autre, par une articulation, l'extrémité supérieure de l'arbre supportant le flotteur.

A l'intérieur du puits, une tablette en fonte est disposée sous l'axe du clapet, elle supporte les ressorts d'ouverture et de fermeture de ce dernier.

Si nous nous reportons aux calculs qui précèdent, nous avons vu que l'effort nécessaire pour l'ouverture dụ clapet et l'admission de l'eau dans l'échelle en période de crue, le puits se remplissant, est de 38 kilogrammètres, et l'effort nécessaire pour la fermeture du clapet, le puits se vidant, est de I $_{2}$ kilogrammètres.

M. Aveque a donc disposé sur la tablette dont il a été parlé ci-dessus deux ressorts, un pour l'ouverture, l'autre pour la fermeture du clapet, main. tenus par des leviers de 85 millimètres de longueur.

La puissance du ressort d'ouverture sera donc de :

$$
38 / 0.085=450 \mathrm{kilogs}
$$

et celle du ressort de fermeture de :

$$
\text { I } 2 / 0.085=\text { I. } .320 \mathrm{kilogs} .
$$

Les leviers, tendant ces ressorts sont prolongés àu delà de l'axe sur lequel ils se meuvent par une tige se trouvant sur le parcours respectif de deux biellettes calćes sur l'axe du clapet et correspondant, l'une à l'ouverture, et l'autre à la fermeture.

En période de crue, quand le mouvement ascensionnel du flotteur est amorcé, il est arrêté par le ressort d'ouverture dont la tige cale la biellelte d'ouverture, tant que l'effort développé est inférieur à la résistance du ressort (38 kilogrammètres).

Quand l'effort dépasse la résistance du ressort, la biellette repousse la tige ci-dessus, l'efface, passe pendant que le ressort reprend sa position initiale et que le flotteur libéré de ce frein achève son mouvement ascensionnel aidé par la pression de l'eau sur le clapet qui s'ouvre, livrant passage à l'eau dans le couloir de l'échelle.

Pour la fermeture du clapet, c'est l'opération inverse qui se prodult, la descente du flotteur sous l'action de son propre poids est arrêtée, tant que la pression exercée par la tige du ressort de fermeture sur la biellette de fermeture est supérieure à l'effort de traction dévcloppé par le flotteur, c'està-dire quand il est encore délesté par une certaine quantité d'eau dans le puits.

Quand l'effort du flotteur est supérieur à la résistance du ressort de fermeture ( I 2 kilogrammètres), la tige de ce dernier s'efface devant la biellette de fermeture, qui se trouve libérée en mème temps que le flotteur auquel elle est fixée par l'intermédiaire de l axe du clapet.

Le flotteur achève donc son mowement de descente et le clapet se ferme lentement, freiné par la pression de l'eau sur sa face amont qui va en 
augmentant jusqu'à ce que l'écart entre les forces antagonistes soit suffisant pour maintenir la fermeture.

Ceci rappelé, le problème se résume à la recherche de la quantité d'eau maximum $Q_{\text {I }}$ ou $Q_{2}$ à admettre dans le puits pour qu'elle interdise le passage de la biellette d owerture devant la tige du ressort d'ouverture.

Suivant les chiffres donnés ci-dessus, l'effort ascensionnel développé par $Q_{\text {I }}$ ou $Q_{2}$ devra ètre inférieur à 38 kilogrammètres et l'effort ascensionnel développé par $Q_{1}+Q_{2}$ derra être supérieur à I 8 kilogrammètres.

Or, cet effort asccnsionnel de 38 kilogrammètres ne peut être atteint et maintenu que si le flotteur est complètement noyé et si la hauteur d'eau admise dans le puils le recouvre encore lorsqu'il est au bout de sa course ascensionnelle.

La longueur de cette course verticale étant de 0.35 pour un flotteur normal, la hauteur d'eau dans le puits, quand le clapet sera maintenu ouvert devra être de :

Cales en bois au fond du puits......... o. 10

Hauteur du flotteur................ 0.90

Course ascensionnelle du flotteur........ 0.35

Hauteur d'eau au-dessus du flotteur pour qu'il

reste noyé $\ldots \ldots \ldots \ldots \ldots \ldots \ldots \ldots \ldots, 0.15$

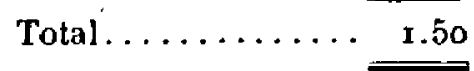

il suffira donc, pour éviter l'amorce du mouvement ascensionnel du flot. teur dans le puits, que la hauleur d'eau à y admeltre ne dépasse pas un mètre, qu'elle vienne de l'aval ou de l'amont.

\section{APPLICATIONS}

\section{A. - barkage de Retenue de caen, sur l'orne (Planche III)}

Si nous appliquons au barrage de retenue de Caen, sur l'Orne, ce qui a été exposé ci-dessus, nous avons les données suivantes (N. G. F.) que nous utiliserons avec étiage normal et crue à l'amont conjuguée avec les basses eaux, mortes eaux, movennes ou ordinajres et hautes eaux vives, eaux moyennes ou ordinaires à l'aval.

Soit niveau légal de retenue......... 7.74

basses eaux mortes, eaux moyènnes... 4.50

$"$ vives, $"$ ordinaires ... 5.20

hautes eaux mortes, eaux moyennes... 6.20

" vives, " 


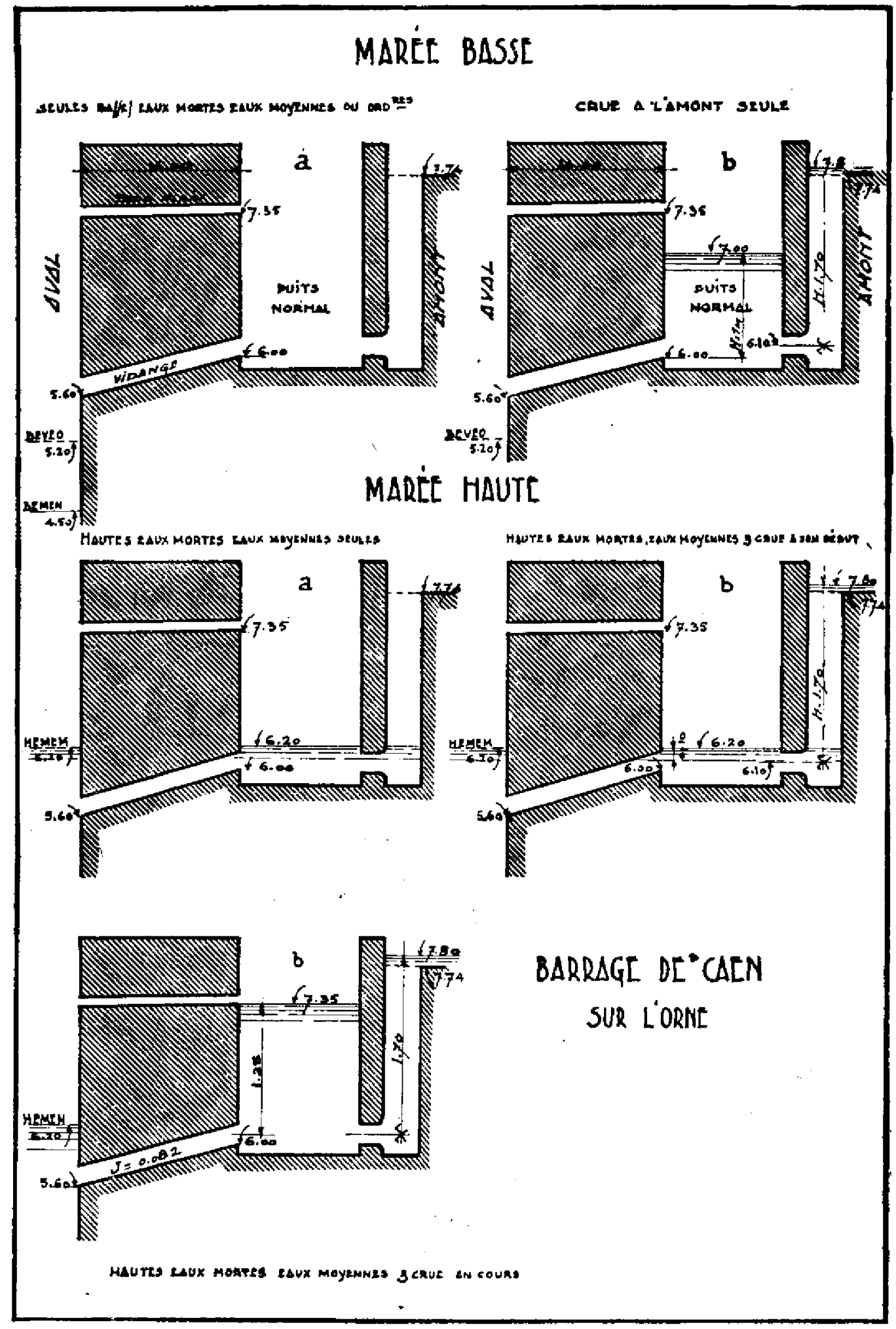

- Plaxche III. - Sehémas de fonctionnement du clapet automatique à Caen. 


\section{I. - MARÉE BASSE}

a) Basses eaux mortes, eaux moyennes ou ordinaires seules

Les cotes des basses eaux mortes caux moyennes (4.50) et des basses éaux vives eaux ordinaires (5.20) étant inférieures à l'orifice aval de la vidange du puits (5.6o), l'eau ne peul, dans ce cas, refouler dans le puits el le clapet reste fermé, le flotteur reposant au fonds du puits.

\section{b) Crue à l'amont seule}

$1^{\circ}$ Alimentation, le puits étant vide. - Si une crue se produit à l'amont ¿a marée basse, l'eau admise dans le puits devra être facilement évacuée afin qu elle ne dépasse par la cote 7.00 , au-delà de laquelle le flotteur se soulèverait.

Si nous appliquons la formule :

$$
\mathrm{Q}=\mathrm{m} \omega \sqrt{2 \mathrm{gH}}
$$

a un orifice d'alimentation du puits de 0.20 de diamètre tel que l'a conçu M. Aveque avec unc crue de 6 centimètres au-dessus du déversoir, nous avons :

$$
\begin{aligned}
& \mathbf{m}=0.80 \\
& \omega=0.10 \times 0.10 \mathrm{~s} \times 3.1416-0.0314 \\
& H=\mathbf{1} .70 \\
& Q=0.80 \times 0.0314 \times 5.77=\mathrm{I} 45 \mathrm{ls}
\end{aligned}
$$

$2^{\circ}$ Alimentation, le puits se remplissant jusqu'à la cote 7.00. - La vidange du puits sera établie de telle sorle qu'elle n'évacuera l'eau provenant d'une crue que si le niveau de l'eau, dans le puits, atteint la cote 7.00 ; à ce moment, le débit de l'alimentation sera réduit à

$$
\begin{gathered}
\mathrm{Q}=\mathrm{m} \omega \sqrt{2 g \mathrm{H}-\mathrm{h}} \\
\text { dans laquelle } \mathrm{m}=0.6 \mathrm{o} \\
\mathrm{H}-\mathrm{h}=7.80-7.00: 0.8 \mathrm{o} \\
\text { soit } \mathrm{Q}=-0.60 \times 0.0314 \times 3.95=75 \mathrm{ls}
\end{gathered}
$$

$3^{\circ}$ Evacuation des 75 litres seconde ci-dessus, le puits étant plein jusqu'à la cole 7.00. - Dans ce cas, la perte de charge de $\mathrm{J}=7.00-5.60=$ I.40/14.00 $=0.10$ centimetres.

Si nous appliquons la formule de DARcy :

$$
\mathrm{x}=\frac{\mathrm{J}}{\mathrm{Q}_{2}} \text { dans laquelle }
$$




$$
X=6.4846 \frac{\mathrm{J} \mathrm{l}}{\mathrm{DS}} \quad \mathrm{Q}=7^{566 \mathrm{ls}}
$$

I I - Coefficient variable avec le diamètre et l'encrassement des tuyaux

$$
x=\frac{0.10}{0.075 \times 0.07^{5}}=17.777
$$

correspondant à un diamètre de o. 8 centimètres. Le diamètre de la vidange du puits sera de 0.18 centimètres.

\section{II. - Marée haute}

\section{a) Hroutes equx mortes eaux moyennes seules}

La cole des hautes eaux mortes eaux moyennes, élant de 6.20 et l'orifice aval de la vidangre du puils étant à la cote 5.60 , le puits se remplira jusquà la cote 6.20 , bien inférieure à la cole nécessaire pour que le flolteur se maintienne soulevé (7.35).

Au reflux, le puits se videra en mème temps que la mer baissera.

\section{b) Hautes eaux mortes eaux moyennes et crues}

Si en hautes eaux mortes eaux moyennes, une crue se produit à l'amont, elle admettra dans le puits contenant lui-meme de l'eau jusqu'à la cote 6.20 par le tuyau de 20 centimètres prévu, une quantité déterminée par la formule :

$$
\begin{gathered}
\mathrm{Q}=\mathrm{m} \omega \sqrt{2 \mathrm{gH}-\mathrm{h}^{\prime}} \\
\text { dans laquelle } \mathrm{m}=0.60 \\
\omega=0.0314 \\
\mathrm{H}-\mathrm{h}^{\prime}=\mathrm{I} .70-0.10=1.60 \\
\text { soit } \mathrm{Q}=0.60 \times 0.03 \mathrm{I} 4 \times 5.60=105 \mathrm{ls}
\end{gathered}
$$

Cette quantité d'eau, provenant de l'amont, fera monter le niveau dans le puits et la cale 7.35 nécessaire pour que le flotteur se soulève sera rapidement atteinte; quand elle la dépassera, le trop plein prévu au puits fonctionnera.

A cet instant, le débit de l'alimentation sera de :

$$
\mathrm{Q}=\mathrm{m} \omega \sqrt{2 \mathrm{~g} \mathbf{H}-\mathbf{h}^{\prime \prime}}
$$

dans laquelle $\mathrm{m}=0.6 \mathrm{o}$

$$
\begin{aligned}
& \mathbf{H}-\mathbf{h}^{\prime \prime}=7.80-7.35=0.45 \mathrm{~d} \text { 'où } \\
& \mathrm{Q}=0.60 \times 0.03 \mathrm{I} 4 \times 2.97=56 \mathrm{ls} .
\end{aligned}
$$

La canalisation d'évacuation de l'eau du puits en 18 centimètres de diamètre évacuera alors, la perte de charge étant de : 


$$
\begin{gathered}
-\mathrm{I} 67- \\
\mathrm{J}=7.35-6.20=\mathbf{1} .15 / \mathbf{1} 4.00=0.082 \\
Q=\sqrt{\frac{0.082}{19.836}}=64 \mathrm{ls} .
\end{gathered}
$$

L'équilibre entre le débit d'admission et l'évacuation sera sensiblement essuré (I).

\section{c) Haules eara vives eaux ordinaires}

Le flotteur sera fatalement noyé en hautes eaux vives eaux ordinaires, l'eau refoulant dans le puits jusqu'à la cote 7.40 .

Le flotteur se soulèvera et le clapet s'ouvrira, mais cela.n'aurait que peu d'importance, mème en étiage normal à l'amont, car l'ouverture du clapet sera brève.

Elle permettra néanmoins de profiter de la remontée des Saumoṇs, plus importante au cours des grandes marées.

La différence entre les étiages amont et aval sera réduite à $7 \cdot 74-7.40=$ o.34 centimètres et l'échelle sera en grande partie noyée, le débit, compte tenu de la hauteur des amortisseurs, serait de r.000 ls environ.

\section{B. - BARRAGE DE RETENUE DE SAINT-BRIEUC, SUR LE GOUET}

(Planche IV)

Ce ibarrage, édifié sur le Gouët, élève le plan d'eau de la rivière à la cote ri.oo à l'amont ; Ie parement aval est battu par la marée suivant les cotes ci-après :
mi-marée $\ldots \ldots \ldots \ldots \ldots \ldots .6 .20$
plus petites mortes eaux..... 7.60
mortes eaux moyennes...... 8.50
plus grandes mortes eaux.... 8.40
plus petites vives eaux...... 10. 10
vives eaux moyennes........ I I .25, Ces dernières cotes dépassent plus grandes vives eaux...... I 2.40 la crête du barrage.

Il faut donc étudier les conditions de fonctionnement du clapet, le puits étant alimenté par l'aval et par l'amont dans les différentes éventualités envisagées pour le barrage de Caen.

Si la cote de remplissage du puits par l'aval est fixée à 8.80 et que le radier soit à 9.20 , les conditions de fonctionnement du clapet à aménagrer

(1) L'obligation de n'utiliser que des sections commerciales ne nous a pas permis de "serrer" de plus près l'équilibre des débits, celui-ci sera obtenu au moyen de vannettes ainsi qu'il est dit plus loin. 


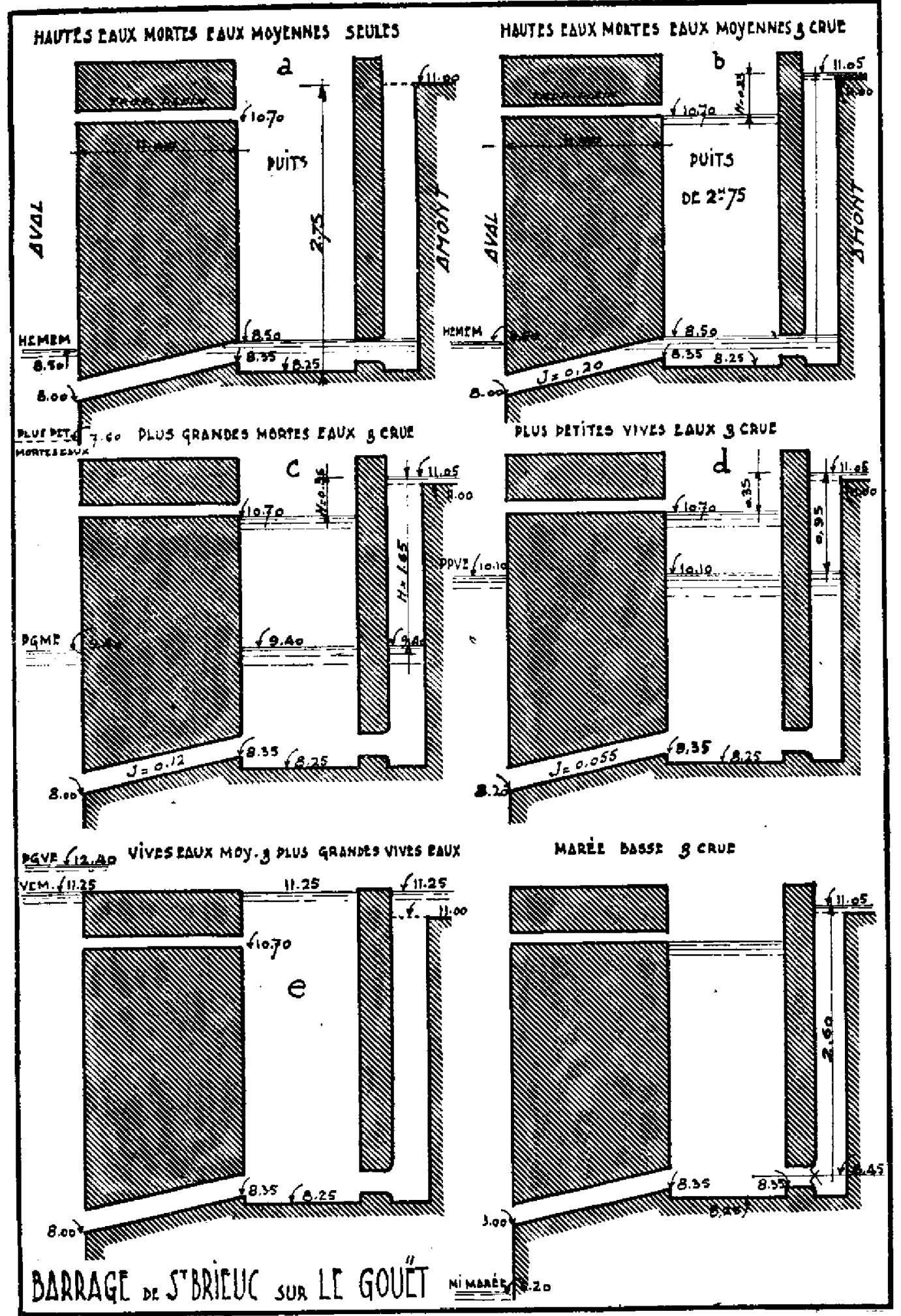

Pianche IV, - Schémas du fonctionnement du clapet automatique à Saint-Brieuc. 
sur le barrage du Gouët seraient identiques aux conditions de fonctionnement au barrage de Caen, les débits d'alimentation et d'évacuation d'eaı étant respectivement identiques à très peu près.

Mais il apparaît que le clapel ne fonctionnera que pendant les plus grandes mortes, eaux et que l'échelle, par conséquent, sera à sec pendant les plus petites mortes eaux $(7,60)$, soit pendant un nombre de jours assex élevé chaque mois.

Il serait donc souhaitable de permettre le fonctionnement du clapel pendant les mortes eaux moyennes. Pour ce faire, il sera nécessaire d'abaisser la cote de remplissage du puits à 8.00 et le radier à 8.25 .

Le puits aura ainsi $2 \mathrm{~m}$. 75 de profondeur et le flotteur, dont le poids et le volume devront être identiques au flotleur type, aura $0.70 \times 0.70$ de section pour 2 mètres de hauteur. Les autres organes du mécanisme ne subiront aucun changement, l'alimentation du puits par l'amont ayant 0.20 de diamètre et l'évacuation étant réduite à 0.12 centimètres de diamètre (ou mème de 0.20 centimètres avec vannette de réglage).

\section{Manée haute}

\section{a) Hautes caux mortes eaux moyennes senles}

La cote des hautes eaux mortes eaux moyennes, étant 8.50 et l'orifice de vidange du puits étant à 8.00 , le puits se remplira jusqu'à la cote 8.50 , bien inférieure à la cote nécessaire pour que le flotteur se soulève (10.70). du reflux, le puits se videra en mème temps que baissera la mer. 楼,

b) Hautes eaux mortes, eaux moyennes et crue.

Si, en haufes eaux mortes, eaux moyennes, une crue de 0.05 centimètres se produit à l'amont, elle introduira dans le puits contenant déjả de l'eau jusqu'à la cote 8.50 la quantité d'eau suivante :

$$
\begin{aligned}
& \mathbf{H}-\mathbf{h}=\mathrm{I} \mathbf{1 . 0 5}-8.50=2.55 \\
& \mathrm{Q}=0.60 \times 0.03 \mathrm{I} 4 \times 7.07=\mathrm{I} 33 \mathrm{Is}
\end{aligned}
$$

qui fera monter le niveau dans le puits. La cote io. jo séra atteinte et le flotteur se soulèvera, entraînant l'ouvcrture du clapet de l'échelle ; ruand la cote ro.7o sera dépassée, le trop plein fonctionnera.

A cet instant, le débit de l'alimentation sera réduit à

$$
\begin{aligned}
& \mathrm{H}-\mathrm{h}=\mathrm{I} \mathrm{I} .05-\mathrm{I} 0.7^{\mathrm{O}}=0.35 \text { centimètres } \\
& \mathrm{Q}=0.60 \times 0.03 \mathrm{I} 4 \times 2.62=49 \mathrm{ls} .
\end{aligned}
$$

L'évacuation, par contre, avec une perte de charge $J$, (la longueur de la canalisation étant de i m mètres) :

$$
J=10.70-8.50=2.20 / 11.00=0.20 \text { centimètres }
$$


correspondra à un débit de 34 ls pour un tuyau de vidange de 0.12 centimètres de diamètre.

c) Phis grandes mortes eaux et crue.

La cote des plus grandes mortes eaux est de 9.40 , l'eau pénètrera dans. le puits jusquà la cole 9.40 , et une crue introduira une quantité pour

$$
\begin{aligned}
& \mathrm{H}-\mathrm{h}=\mathrm{1} \mathrm{1.05}-\mathrm{g} .40=\mathrm{r} .65 \\
& \mathrm{Q}=0.60 \times 0.0314 \times 5.68=106 \mathrm{ls}
\end{aligned}
$$

quand la cote ro.7o sera atteinte dans le puits pour

$$
\begin{aligned}
& \mathbf{H}-\mathbf{h}=\mathbf{I} \mathbf{I} .05-10.7 \mathrm{o}=0.35 \\
& \mathrm{Q}=49 \mathrm{ls} .
\end{aligned}
$$

L'évacuation débitera avec un tuyau de o.1' pour

$$
\mathrm{J}=10.70-9.40=1.30 / 1 \mathrm{I}=0.12
$$

une quantité de $27 \mathrm{ls}$.

d) Plus petites vives eaux et crues.

La cote des plus petiles vives eaux est de 10.10 et l'cau péuètrera dans le puits à la cote ro. ro, uné crue introduira dans le puits pour

$$
\begin{aligned}
& H-h=11.05-10.10=0.95 \\
& Q=0.60 \times 0.0314 \times 4.31=81 \mathrm{ls}
\end{aligned}
$$

quand la cole 10.70 sera maintenue dans le puits pour

$$
\mathbf{H}-\mathbf{h}=\mathbf{I} \mathbf{1 . 0 5}-10.70=0.35 \text { on aura } \mathrm{Q}=49 \mathrm{ls} \text {. }
$$

L'évacuation débitera, à ce moment, avec un tuyau de o. I 2 pour

$$
\mathrm{J}=10.70-10.10=0.60 / 1 \mathrm{I}=0.055
$$

une quantité de 19 ls.

e) Vives eaux moyennes et plus grandes vives eaux et crue

Au cours de ces marées, le barrage est recouvert, l'échelle et le méca. nisme du clapet sont complètement novés et la remontée des migrateurs se fait sans aucun obstacle.

\section{Marée basse}

A marée basse, si une crue de o.ō vient à se produire en amont, l'alimentation du puits débitera, pour

$$
\begin{aligned}
& \mathrm{H}-\mathrm{h}=\mathrm{I} 1.05-8.45-2.60 \quad \mathrm{~m}=0.80 \\
& \mathrm{Q}=0.80 \times 0.0314 \times 7.14=179 \mathrm{ls} .
\end{aligned}
$$

Le niveau de l'eau montera dans le puits jusqu'à la cote Io.70, le flot- 
teur se soulèvera en ouvrant le clapet, et l'échelle fonctionnera, car le débit de la vidange sera pour.

$$
\begin{gathered}
J=10.70-8.00=2.70 / 11.00-0.25 \\
\operatorname{de} \sqrt{\frac{0.25}{160}}=401 \mathrm{~s}
\end{gathered}
$$

en conséquence, le trop plein déversera le surplus de l'alimentation sur le débit de l'évacuation.

\section{CONCLUSIONS}

L'exposé qui précède permet donc d'envisager l'installation du clapet automatique Aveque avec de légères modiftcations, sur les échelles à pois. sons des barrages soumis à l'action des marées. Les sections d'admission et de vidange pourraient être conservées telles que M. Aveque les a calculées, mais il serait prudent de les aug̣menter afin d'éviler leur obstruction, le reglage minutieux des débits d'alimentation (amont et aval) et de vidange par l'aval étant obtenu au moyen de deux vannettes.

L'admission d'eau de mer à l'aval devra présenter un dispositif analogue au dispositif d'admission à l'amont. Des précautions seront prises pour éviter l'entrée dans le puits des débris et épaves apportés par le flux.

Quand on se trouvera en présence d'amplitude de marée considérable, telle qu'on en observe sur le littoral Nord de la Bretagne, le puits devra être approfondi afin de permettre la mise cn service de l'échelle, même pendant unc partie des hautes eaux mortes eaux. La cote de remplissage du puits par l'aval, qui déterminera la durée d'ouverture du clapet, ainsi que la cote inférieure de l'échclle devnont ĉtre soigneusement déterminées. en raison de lamplitude variable des marées pour chaque point du littoral.

Les indicatioṇs nécessaires pourront être recueillies au Service hydrographique de la Marine.

Par ailleurs, les parties métalliques de l'échelle, éléments amortisseurs, flotteur, clapet, etc..., en tôle, susceptibles d'être en contact avec l'eau de mer, devront être parfaitement entretenues de peinture afin d'éviter leur corrosion. Partout où cela sera possible, l'emploi de Ia fonte sera recommandé, même pour la fabrication des paliers.

Il sera prudent de remplacer les ressorts d'ouverture et de fermeture, particulièrement fragriles, et dont la corrosion modifierait la résistance, far deux contrepoids, reliés à l'axe du clapet par un systèrne de bielles logées dons le puits agrandì à cel effet ou par des ressorts en cuivre.

Enfin, les canalisations en ciment seront avantageusement remplocées par des tuyaux en grés vernissé, et l'emploi de ciment alumiñeux fondu résistant à l'eau de mer est recommandé. 